\title{
Associação entre absenteísmo e ambiente de trabalho dos técnicos de enfermagema
}

\author{
Association between absentism and work environment of nursing technicians \\ Asociación entre absentismo y entorno de trabajo de técnicos de enfermería
}

Priscila Braga de Oliveira ${ }^{1}$ (D) Letícia Nunes $\mathrm{Coca}^{1}$ (1) Wilza Carla Spiri ${ }^{1}$ (1)

1. Universidade Estadual "Júlio de Mesquita Filho", Faculdade de Medicina de Botucatu. Botucatu, SP, Brasil.
Autor Correspondente:

Priscila Braga de Oliveira.

E-mail: pboliveira00@gmail.com.

Recebido em 22/06/2020.

Aprovado em 30/09/2020

\section{RESUMO}

Objetivo: analisar se o ambiente de trabalho e as características sociodemográficas e laborais influenciam o absenteísmo de técnicos de enfermagem. Método: estudo transversal e correlacional, em unidade de urgência e emergência com técnicos de enfermagem. Aplicado instrumento com dados sociodemográficos e laborais, absenteísmo referido e versão brasileira validada para técnicos de enfermagem do Nursing Work Index Revised (B-NWI-R). Resultados: participaram 62 técnicos de enfermagem, predominância do sexo feminino, jovens, trabalho de 36 a 40 horas semanais e turno diurno. Absenteísmo fo referido por $33,87 \%$ dos participantes e o ambiente de trabalho foi considerado favorável à prática, com média 2,47. A cada ponto na escala B-NWI-R a chance de absenteísmo aumenta em 2,63 vezes, ou seja, quanto mais desfavorável à prática profissional é o ambiente de trabalho maior é a chance de absenteísmo. Conclusão: um ambiente de trabalho desfavorável à prática profissional aumenta a chance de absenteísmo entre os técnicos de enfermagem. Implicações para a prática: uma análise do absenteísmo associada ao ambiente de trabalho dos técnicos de enfermagem contribui para que os gerentes de enfermagem criem estratégias para melhorar o ambiente de trabalho.

Palavras-Chave: Absenteísmo; Ambiente de Instituições de Saúde; Equipe de Enfermagem; Enfermagem; Emergência.

\begin{abstract}
Objective: to analyze whether the work environment and socio-demographic and work characteristics influence the absenteeism of nursing technicians. Method: a cross-sectional and correlational study, conducted in an urgency and emergency unit with nursing technicians. Instrument applied with sociodemographic and work data, referred absenteeism and validated Brazilian version for nursing technicians of the Nursing Work Index Revised (B-NWI-R). Results: a total of 62 nursing technicians participated, predominantly female, young, working from 36 to 40 hours a week and day shift. Absenteeism was reported by $33.87 \%$ of the participants and the work environment was considered favorable to the practice, with a mean of 2.47 . At each point on the B-NWI-R scale, the chance of absenteeism increases by 2.63 times, that is, the more unfavorable the professiona practice is to the work environment, the greater the chance of absenteeism. Conclusion: a work environment unfavorable for the professional practice increases the chance of absenteeism among nursing technicians. Implications for the practice: an analysis of absenteeism associated with the work environment of nursing technicians helps nursing managers to create strategies to improve the work environment.
\end{abstract}

Keywords: Absenteeism; Environment of Health Institutions; Nursing team; Nursing; Emergency.

\section{RESUMEN}

Objetivo: analizar si el entorno laboral y las características sociodemográficas y laborales influyen en el absentismo de los técnicos de enfermería. Método: estudio transversal y correlacional, en una unidad de urgencias y emergencias con técnicos de enfermería. Instrumento aplicado con datos sociodemográficos y laborales, ausentismo referido y versión brasileña validada para técnicos de enfermería del Nursing Work Index Revised (B-NWI-R). Resultados: participaron 62 técnicos de enfermería, predominantemente mujeres, jóvenes, trabajando de 36 a 40 horas a la semana y turno de día. El ausentismo fue reportado por el $33,87 \%$ de los participantes y el ambiente de trabajo se consideró favorable a la práctica, con un promedio de 2,47 . En cada punto de la escala B- NWI-R, la posibilidad de absentismo aumenta en 2,63 veces, es decir, cuanto más desfavorable sea la práctica profesional en el entorno laboral, mayores serán las posibilidades de absentismo. Conclusión: un ambiente de trabajo desfavorable para la práctica profesional aumenta las posibilidades de absentismo entre los técnicos de enfermería. Implicaciones para la práctica: un análisis del absentismo asociado con el ambiente de trabajo de los técnicos de enfermería ayuda a los gerentes de enfermería a crear estrategias para mejorar el ambiente de trabajo.

Palabras Claves: Ausentismo; Entorno de Instituciones de Salud; Equipo de Enfermería; Enfermería; Emergencia 


\section{INTRODUÇÃO}

A enfermagem insere-se como trabalho complexo no contexto da saúde, sendo necessário para seu desempenho uma equipe adequadamente dimensionada e qualificada inserida no ambiente de instituições de saúde.

O absenteísmo é o termo utilizado para indicar o ato de um trabalhador não comparecer ao trabalho em um período designado a ele. ${ }^{1,2}$ É de etiologia multifatorial, podendo ser dividido em três áreas: fatores pessoais, como idade, características familiares e qualidade de vida; fatores organizacionais, como satisfação com o trabalho e com a liderança; e fatores relacionados ao local de trabalho, como sobrecarga de atividades e qualidade do ambiente laboral. ${ }^{3,4}$

Identificar a influência direta da qualidade do ambiente de trabalho sobre o absenteísmo é imprescindível, já que esse conhecimento fornece subsídios para a gerência das instituições de saúde promover ações que resultem na melhoria da qualidade do ambiente, seja com melhorias de infraestrutura, de dimensionamento de recursos humanos e de cultura organizacional; para que assim a saúde do trabalhador não seja impactada, aumentando a satisfação profissional, reduzindo o absenteísmo e consequentemente melhorando a qualidade da assistência.

O ambiente de trabalho é fator inerente à satisfação da equipe de enfermagem e ao desempenho adequado da prática profissional. A sobrecarga de trabalho, a pouca autonomia, a ambiguidade de funções, a presença de relações conflituosas e a falta de expectativa profissional caracterizam um ambiente de trabalho desfavorável. ${ }^{5-7}$

Já o cuidado focado na qualidade, o apoio ao desenvolvimento profissional, a colaboração entre diferentes profissionais da saúde, a promoção da liderança e da autonomia da enfermagem favorecem o estabelecimento de um ambiente favorável à prática, que propicia a obtenção de melhores resultados não só para o paciente, mas também para a equipe e para a instituição, já que um ambiente de trabalho positivo impacta na eficácia do próprio trabalho..$^{5,7,8}$

Assim, é fundamental que esse ambiente de trabalho tenha profissionais de enfermagem envolvidos com o processo de cuidado para a garantia da assistência segura.

Os serviços hospitalares de urgência e emergência representam um ambiente de alta rotatividade de pacientes, que exigem eficiência e rapidez na realização de procedimentos, a fim de manter a vida dos indivíduos, que apresentam sinais e sintomas imprevisíveis, além de elevado risco de morte..$^{9,10}$

$A$ atuação da equipe de enfermagem, nesse ambiente específico, pode contribuir para exposição a riscos ocupacionais, devido à sua dinamicidade, heterogeneidade, proximidade física ao paciente e manuseio de materiais e equipamentos; o que pode acarretar o aumento do absenteísmo dos profissionais e a diminuição da qualidade da assistência. ${ }^{10,11}$

Os técnicos de enfermagem representam $77 \%$ dos profissionais de enfermagem no Brasil. ${ }^{12}$ Esse profissional, segundo a Lei do Exercício Profissional (№ 7.498/86), exerce atividades de nível médio, como ações assistenciais, de orientação e supervisão do trabalho de enfermagem de forma auxiliar, podendo desempenhar as suas funções somente sob orientação e supervisão do Enfermeiro. ${ }^{13}$

Estudo ${ }^{14}$ demonstrou que quanto menor o nível hierárquico do trabalhador maior o número de afastamentos por doença, destacando-se assim que o técnico de enfermagem se afasta mais do trabalho que os enfermeiros.

Considerando que os técnicos de enfermagem atuam em todos os cenários de assistência à saúde no desenvolvimento do cuidado, encontrando-se na linha de frente para assistir os usuários desses cenários, é imprescindível conhecer no cenário das urgências e emergências como se dá essa atuação.

Assim, infere-se que o absenteísmo é influenciado pelo ambiente de trabalho dos profissionais de enfermagem, no entanto, embora haja abundante literatura de estudos acerca do absenteísmo, há escassez sobre como se articula com o ambiente de trabalho, em especial como se dá essa relação com os técnicos de enfermagem, justificando assim o presente estudo. Portanto, a finalidade do estudo é conhecer essa relação e contribuir para melhoria do ambiente de trabalho e a redução do absenteísmo.

Considerando a questão: "Quais são os fatores associados ao absenteísmo entre os técnicos de enfermagem de uma unidade de urgência e emergência?" o objetivo do estudo foi analisar se as características do ambiente de trabalho e as características sociodemográficas e laborais influenciam o absenteísmo de técnicos de enfermagem de uma unidade de urgência e emergência.

\section{MÉTODO}

Estudo transversal e correlacional, realizado na unidade de urgência e emergência de um hospital público terciário do Estado de São Paulo referência da Rede de Atenção à Saúde 9.

A amostra, não probabilística por conveniência, foi constituída de 62 técnicos de enfermagem, o que correspondeu à totalidade dos trabalhadores da unidade, que concordaram em participar da pesquisa após esclarecimentos e garantia do anonimato.

Foi utilizado o seguinte critério de inclusão: ser técnico de enfermagem em atividade na unidade de urgência e emergência. Os critérios de exclusão foram: profissionais que estavam de licença saúde prolongada, licença maternidade ou desistência da participação durante a coleta de dados.

$O$ instrumento para análise de ambiente de trabalho foi o Nursing Work Index Revised na versão brasileira (B-NWI-R). Trata-se de uma escala de medida do tipo Likert, cuja pontuação varia entre um a quatro pontos. O B-NWI-R para técnicos de enfermagem é uma escala com 15 itens com as mesmas subescalas da validada para enfermeiros: autonomia; controle sobre o ambiente; relação entre médicos e equipe de enfermagem e suporte organizacional. ${ }^{15}$

O participante que se inseriu no critério de inclusão do estudo foi convidado a responder a escala B-NWI-R, se concordava ou não com a afirmativa "esse fator está presente no meu trabalho 
diário" com as opções: concordo totalmente (um ponto); concordo parcialmente (dois pontos); discordo parcialmente (três pontos) e discordo totalmente (quatro pontos), ou seja, quanto menor a pontuação, maior a presença de atributos favoráveis. Valores abaixo de 2,5 representam ambientes favoráveis à prática profissional, e acima de 2,5 pontos, ambientes desfavoráveis. ${ }^{16}$

Os dados sociodemográficos e laborais foram constituídos das variáveis: sexo, idade, etnia, estado civil, número de filhos, categoria profissional, horas de trabalho semanal, trabalho em unidades cuja permanência do paciente é superior a cinco dias, promoção no último ano, avaliação de desempenho profissional, turno de trabalho, trabalho em horário denominado administrativo (oito horas por dia no período de segunda a sexta-feira), presença de outro emprego formal, tempo de trabalho na instituição e satisfação com o salário.

O absenteísmo referido foi obtido a partir de uma pergunta no questionário sociodemográfico e laboral onde o participante descrevia se havia ou não se ausentado do trabalho, se a ausência foi justificada ou não, qual o motivo e quantos dias ficou ausente.

A escala B-NWI-R e o questionário foram aplicados no mês de janeiro de 2018, com o objetivo de obter dados referentes ao ano de 2017. A coleta de dados ocorreu na unidade de urgência e emergência referida, em um ambiente calmo, uma sala sem ruídos extenos. A duração média do preenchimento dos dados foi de 15 minutos por participante. Salienta-se que o pesquisador esteve presente durante toda a aplicação do questionário e esclareceu as dúvidas referentes ao instrumento quando solicitado.
A análise da chance de absenteísmo em função do ambiente de trabalho medido pela escala B-NWI-R foi realizada por meio de modelo de regressão logística múltipla incluindo os dados sociodemográficos e laborais como potenciais confundidores. Associações foram consideradas estatisticamente significativas se $\mathrm{p}<0,05$.

A análise da chance de absenteísmo e o cálculo do coeficiente de alfa de Cronbach, para a obtenção da confiabilidade da escala B-NWI-R foram realizados com o software Statistical Package for the Social Sciences - SPSS 21.

O projeto foi encaminhado e aprovado pelo Comitê de Ética em Pesquisa (Parecer no. 2.076.259).

Os técnicos de enfermagem e os enfermeiros da Unidade de Urgência e Emergência foram convidados a participar do estudo garantindo o anonimato e o esclarecimento de todas as etapas da pesquisa. Aos que aceitaram participar foi solicitada a assinatura do Termo de Consentimento Livre e Esclarecido.

\section{RESULTADOS}

Os participantes do estudo apresentaram as seguintes características: maioria de mulheres, faixa etária de 31 a 40 anos, brancos e casados.

Em relação aos dados laborais, trabalhavam em jornadas de 30 a 40 horas semanais, no turno diurno, de um a cinco anos na instituição, não estavam satisfeitos com o salário e a maioria possuía somente nível técnico (Tabela 1).

Tabela 1. Dados demográficos e laborais dos Técnicos de Enfermagem da Unidade de Urgência e Emergência ( $n=62)$. Botucatu, 2018.

\begin{tabular}{|cc}
\hline \multicolumn{1}{|c}{ Variável } & $\mathbf{N}(\%)$ \\
\hline Sexo & \\
\hline Sexo feminino & $55(88,7)$ \\
\hline Sexo masculino & $7(11,3)$ \\
\hline Idade & $13(29,9)$ \\
\hline 20 a 30 anos & $23(37,1)$ \\
\hline 31 a 40 anos & $17(27,5)$ \\
\hline 41 a 50 anos & $9(14,5)$ \\
\hline Acima de 50 anos & \\
\hline Etnia & $43(69,4)$ \\
\hline Branca & $13(20,9)$ \\
\hline Parda & $06(9,7-)$ \\
\hline Preta & \\
\hline Estado Civil & $15(24,2)$ \\
\hline Solteiro & $37(59,6)$ \\
\hline Casado & $10(16,2)$ \\
\hline Divorciado & - \\
\hline Viúvo & \\
\hline Jornada de Trabalho & \\
\hline
\end{tabular}


Tabela 1. Continuação...

\begin{tabular}{|c|c|}
\hline Variável & $N(\%)$ \\
\hline $30 h$ & $29(46,8)$ \\
\hline 36 & $2(3,2)$ \\
\hline $40 \mathrm{~h}$ & $27(43,5)$ \\
\hline Dupla jornada & $4(6,5)$ \\
\hline \multicolumn{2}{|c|}{$\begin{array}{l}\text { Trabalho em Unidade com pacientes internados acima de } \\
5 \text { dias }\end{array}$} \\
\hline Sim & $47(75,8)$ \\
\hline Não & $15(24,2)$ \\
\hline \multicolumn{2}{|l|}{ Promoção nos últimos 12 meses } \\
\hline Sim & $1(1,6)$ \\
\hline Não & $61(98,4)$ \\
\hline \multicolumn{2}{|c|}{ Avaliação negativa desempenho profissional } \\
\hline Sim & $9(14,5)$ \\
\hline Não & $53(85,5)$ \\
\hline \multicolumn{2}{|l|}{ Turno de Trabalho } \\
\hline Diurno & $36(58)$ \\
\hline Noturno & $25(40,4)$ \\
\hline Diurno e noturno & $1(1,6)$ \\
\hline \multicolumn{2}{|l|}{ Outro emprego formal } \\
\hline Sim & $9(14,5)$ \\
\hline Não & $52(83,9)$ \\
\hline Não informado & $1(1,6)$ \\
\hline \multicolumn{2}{|l|}{ Tempo de Trabalho } \\
\hline$<1$ ano & $4(6,5)$ \\
\hline 1 a 5 anos & $33(53,3)$ \\
\hline 6 a 10 anos & $12(19,3)$ \\
\hline 11 a 15 anos & $2(3,2)$ \\
\hline 16 a 20 anos & $9(14,5)$ \\
\hline$>20$ anos & $1(1,6)$ \\
\hline Não informado & $1(1,6)$ \\
\hline \multicolumn{2}{|l|}{ Satisfação com o salário } \\
\hline Sim & $18(29)$ \\
\hline Não & $43(69,4)$ \\
\hline Não informado & $1(1,6)$ \\
\hline \multicolumn{2}{|l|}{ Nível de formação } \\
\hline Técnico & $60(96,8)$ \\
\hline Nível superior & $2(3,2)$ \\
\hline
\end{tabular}

A ausência ao trabalho no ano de 2017 foi referida pelos profissionais, 21 (33,87\%) faltaram ao trabalho, sendo que $13(62 \%)$ justificaram terem apresentado alguma doença que incapacitou o trabalho, cinco (23,9\%) receberam licença médica, um (4,7\%) sofreu acidente de trabalho, um (4,7\%) sofreu acidente que resultou em politraumatismo e um $(4,7 \%)$ relatou doença em membro da família.
O absenteísmo referido teve média de 42 dias por doença, 111 dias por licença médica, 25 dias por acidente de trabalho, 150 dias por acidente que resultou em politraumatismo e um dia por doença na família.

A escala B-NWI-R aplicada aos técnicos de enfermagem evidenciou a média geral menor que 2,5, no entanto a subescala 
Controle do Ambiente apresentou média acima deste valor conforme demonstrado na Tabela 2.

A confiabilidade da escala B-NWI-R foi analisada através do coeficiente de alfa de Cronbach, para o total

Tabela 2. Escala B-NWI-R e as subescalas: controle sobre o ambiente, autonomia, relação médico-enfermeiro e suporte organizacional dos técnicos de enfermagem $(n=62)$. Botucatu, 2018.

\begin{tabular}{lcc}
\hline Instrumento de Ambiente de Trabalho & Média & DP \\
\hline B-NWI-R- Geral & 2,47 & 0,52 \\
\hline B-NWI-R- Controle Ambiente & 2,68 & 0,56 \\
B-NWI-R- Autonomia & 2,26 & 0,62 \\
\hline B-NWI-R- Relação Médico-Enfermeiro & 2,31 & 0,65 \\
B-NWI-R- Suporte Organizacional & 2,46 & 0,54 \\
\hline
\end{tabular}

B-NWI-R: Nursing Work Index - Revised - Versão brasileira. de itens do instrumento e para cada uma das subescalas, resultando em 0,86 para a escala geral, 0,68 para a subescala Controle sobre o Ambiente, 0,69 para Autonomia, 0,71 na Relação Médico-Enfermeiro e 0,80 para a subescala Suporte Organizacional.

A análise da chance de absenteísmo em função do ambiente de trabalho, medido pela escala B-NWI-R, incluindo os dados sociodemográficos e laborais como potenciais confundidores, não resultou em significância estatística $(p<0,05)$ frente a nenhum fator. Para a escala B-NWI-R, o $p$ foi de 0,077 , enquanto o odds ratio (OR), que indica a razão de chance ou possibilidade, resultou em 2,63. (Tabela 3)

\section{DISCUSSÃO}

O estudo possibilitou a associação entre dados sócio demográficos laborais, absenteísmo e ambiente de trabalho dos técnicos de enfermagem de uma unidade de urgência e emergência.

Tabela 3. Modelo máximo para a chance de absenteísmo entre Técnicos de Enfermagem (n=62). Botucatu, 2018.

\begin{tabular}{|c|c|c|c|c|}
\hline \multirow{2}{*}{$\begin{array}{ll} & \text { Variável } \\
\text { Homens } & \end{array}$} & \multirow{2}{*}{$\begin{array}{c}\text { OR } \\
0,76\end{array}$} & \multicolumn{2}{|c|}{ IC95\% } & \multirow{2}{*}{$\frac{\mathbf{p}}{0,754}$} \\
\hline & & 0,13 & 4,28 & \\
\hline \multicolumn{5}{|l|}{ Idade } \\
\hline 20 a 30 anos & & & & 0,628 \\
\hline 31 a 40 anos & 2,56 & 0,55 & 11,85 & 0,228 \\
\hline 41 a 50 anos & 1,39 & 0,26 & 7,30 & 0,698 \\
\hline Acima de 50 anos & 1,67 & 0,25 & 11,07 & 0,597 \\
\hline \multicolumn{5}{|l|}{ Etnia } \\
\hline Branca & & & & 0,117 \\
\hline Negra & 0,34 & 0,07 & 1,74 & 0,194 \\
\hline Parda & 3,73 & 0,61 & 22,80 & 0,154 \\
\hline \multicolumn{5}{|l|}{ Estado civil } \\
\hline Sem companheiro & 1,58 & 0,54 & 4,58 & 0,403 \\
\hline Tem filhos & 1,20 & 0,32 & 4,46 & 0791 \\
\hline \multicolumn{5}{|l|}{ Jornada de trabalho } \\
\hline 40 horas semanais ou dupla jornada & 0,35 & 0,12 & 1,06 & 0,064 \\
\hline Trabalho em unidades com paciente internado +5 dias & 1,03 & 0,30 & 3,54 & 0,960 \\
\hline Recebeu alguma promoção & $3, E+09$ & 0,00 & & 1,000 \\
\hline Turno noturno (Ref: Diurno) & 1,17 & 0,40 & 3,41 & 0,771 \\
\hline \multicolumn{5}{|l|}{ Horário Administrativo } \\
\hline Sim (Ref: Não) & 2,00 & 0,12 & 33,66 & 0,630 \\
\hline Tem outro emprego & 0,50 & 0,09 & 2,64 & 0,411 \\
\hline Tempo de trabalho (Ref: $<1 \mathrm{ano})$ & & & & 0,752 \\
\hline 1 a 5 anos & $1, \mathrm{E}+09$ & 0,00 & & 0,999 \\
\hline 6 a 10 anos & $8, E+08$ & 0,00 & & 0,999 \\
\hline Acima de 10 anos & $5, E+08$ & 0,00 & & 0,999 \\
\hline Satisfeito com o salário & 0,93 & 0,29 & 2,99 & 0,907 \\
\hline B-NWI-R geral & 2,63 & 0,90 & 7,69 & 0,077 \\
\hline
\end{tabular}


O perfil sociodemográfico dos participantes foi de profissionais mulheres, de idade menor que 35 anos, casadas e de etnia branca, o que é corroborado por estudo realizado pelo Conselho Federal de Enfermagem (Cofen), que demonstrou que $86 \%$ dos profissionais de enfermagem são do sexo feminino, $52 \%$ na faixa etária de até 35 anos, $43,6 \%$ são casados e $57,9 \%$ se consideram de etnia branca. ${ }^{17}$

A jornada de trabalho de 36 a 40 horas semanais, o turno único de trabalho e menos de cinco anos de atividade profissional predominaram na população, concordando com o perfil nacional, no qual $41,5 \%$ têm carga horária de trabalho semanal de 31 a 40 horas, $71 \%$ trabalham no período diurno e $36,5 \%$ têm menos de cinco anos de trabalho na área. ${ }^{17}$

O motivo das ausências entre os profissionais foi majoritariamente devido a doenças, o que foi constatado em estudo realizado no sul do Brasil, que identificou a alta prevalência de absenteísmo por doença na enfermagem, dentre a amostra abordada $36 \%$ dos técnicos de enfermagem se afastaram do trabalho devido a algum problema de saúde. ${ }^{18}$

Apesar de não ter sido referido quais as doenças que desencadearam a ausência ao trabalho, a literatura afirma que os problemas relacionados ao sistema osteomuscular predominam, já que a sobrecarga de trabalho, a longa permanência em pé e a postura corporal incorreta desencadeiam danos à saúde. 18,19

Além disso, enfermidades como depressão, estresse, ansiedade e burnout também tem alta incidência entre os trabalhadores de enfermagem que se ausentam ao trabalho. ${ }^{3,20}$ Esses transtornos são desencadeados, devido à própria natureza do trabalho, principalmente em serviços de urgência e emergência, área na qual o profissional tem que manter pleno controle de situações de extrema vulnerabilidade, o que contribui para o esgotamento físico e mental desse profissional. ${ }^{9}$

Estudo realizado em hospital público universitário do estado de São Paulo relacionou o ambiente de trabalho à qualidade de vida do profissional de enfermagem, constatou que diferentes fatores do ambiente de trabalho, como limitação de recursos, desmotivação e superlotação de pacientes, influenciam na qualidade de vida do trabalhador nos domínios físico, psicológico e também social. ${ }^{7}$

As médias da escala B-NWI-R demonstraram que os técnicos de enfermagem consideraram o ambiente de trabalho da unidade de urgência e emergência favorável. No entanto, quando as subescalas foram analisadas isoladamente, a subescala Controle sobre o Ambiente foi a única cuja média mostrou-se maior que 2,5, portanto desfavorável. Esse resultado é corroborado por estudo realizado em unidade de urgência e emergência na região sul do Brasil. ${ }^{21}$

Estudo realizado em dois hospitais públicos, um acreditado e outro não, revelou que os profissionais de enfermagem de ambos hospitais consideram o ambiente favorável. Outro estudo que adaptou e validou a escala B-NWI-R apresentou resultados semelhantes com a média das subescalas abaixo de 2,5, ressaltando tratar-se de ambiente favorável. ${ }^{5,15}$
A escala se mostrou confiável, com alfa de Cronbach 0,86 para B-NWI-R geral, ou seja, constatou-se que os itens são homogêneos e que a escala mede a característica para a qual foi criada de forma consistente. Outros estudos confirmam esses achados. ${ }^{5,8}$

A partir da análise dos dados foi possível estabelecer uma relação entre o ambiente de trabalho e a chance de absenteísmo de forma quantitativa. O odds ratio (OR), que consiste na razão de chance ou razão de possibilidade, para a escala permite afirmar que quanto mais desfavorável o ambiente, maior a chance de um técnico de enfermagem não comparecer ao trabalho em período designado a ele. Além disso, quantifica que a cada ponto na escala B-NWI-R, a chance de absenteísmo aumenta em 2,63 vezes.

A relação entre a qualidade do ambiente de trabalho e o absenteísmo foi identificada em estudo sul africano, o qual afirmou que profissionais que trabalham em ambientes mais favoráveis à prática, têm a tendência de se ausentar menos ao trabalho, do que indivíduos que trabalham em ambientes físico e psicologicamente exaustivos. ${ }^{22}$

Destacam-se como limitações do estudo o absenteísmo ter sido referido pelos próprios técnicos de enfermagem e o instrumento de coleta de dados ter sido autoaplicado, assim em ambos os casos a honestidade nas respostas foi de responsabilidade exclusiva dos participantes da pesquisa. Além disso, o estudo foi realizado em um único hospital, em um setor específico.

Assim, é fundamental que haja investimento das organizações de saúde na melhoria do ambiente de trabalho. Estudo revela que os gestores são essenciais para instituírem condições que assegurem ao trabalhador da enfermagem o crescimento profissional, a possibilidade de satisfação com o trabalho e o compromisso com a qualidade do cuidado. ${ }^{23}$

\section{CONCLUSÃO}

A elaboração dessa pesquisa permitiu estabelecer uma relação entre a qualidade do ambiente de trabalho e o absenteísmo referido pelos técnicos de enfermagem. A cada ponto na escala BNWI-R a chance de absenteísmo aumenta em 2,63 vezes, ou seja, quanto mais desfavorável à prática profissional é o ambiente de trabalho maior é a chance de absenteísmo. Identificar a relação direta entre a qualidade do ambiente de trabalho e o absenteísmo, além das suas principais causas, fornece subsídios à gerência das instituições de saúde para que invistam em estratégias de melhorias de infraestrutura, de dimensionamento de recursos humanos e de cultura organizacional para que $o$ ambiente se torne mais favorável à prática, reduzindo a incidência de ausências e elevando a qualidade da assistência prestada.

\section{FINANCIAMENTO}

Concedido pela Fundação de Amparo a Pesquisa do Estado de São Paulo - FAPESP - Processo 2017/17710-1, ao projeto de pesquisa regular "Gerenciamento baseado em evidências: 
instrumentos para operacionalização no contexto da enfermagem", beneficiário Wilza Carla Spiri.

\section{CONTRIBUIÇÕES DOS AUTORES}

Desenho do estudo. Priscila Braga de Oliveira, Wilza Carla Spiri. Coleta ou produção dos dados. Priscila Braga de Oliveira. Letícia Nunes Coca.

Análise de dados. Priscila Braga de Oliveira. Letícia Nunes Coca.

Interpretação dos resultados. Priscila Braga de Oliveira. Wilza Carla Spiri. Letícia Nunes Coca.

Redação e revisão crítica do manuscrito. Priscila Braga de Oliveira. Wilza Carla Spiri. Letícia Nunes Coca.

Aprovação da versão final do artigo. Priscila Braga de Oliveira, Letícia Nunes Coca. Wilza Carla Spiri.

Responsabilidade por todos os aspectos do conteúdo e a integridade do artigo publicado. Priscila Braga de Oliveira, Letícia Nunes Coca. Wilza Carla Spiri.

\section{EDITOR ASSOCIADO}

\section{Gerson Luiz Marinho}

\section{REFERÊNCIAS}

1. Lima SRR, Cortez EA, Carmo TG, Soares GRS, Gama LN. Absenteeism with focus on the health of the nursing team worker acting in intensive care: integrative review. J Nurs UFPE on line. 2016;10(9):3426-37. https://doi.org/10.5205/1981-8963-v10i9a11425p3426-3437-2016.

2. Dhaini S, Zúñiga F, Ausserhofer D, Simon M, Kunz R, De Geest S et al. Absenteeism and presenteeism among care workers in swiss nursing homes and their association with psychosocial work environment: a multi-site cross-sectional study. Gerontology. 2016;62(4):386-95. http:// dx.doi.org/10.1159/000442088. PMid:26618789.

3. Ticharwa M, Cope V, Murray M. Nurse absenteeism: an analysis of trends and perceptions of nurse unit managers. J Nurs Manag. 2019 jan;27(1):109-16. http://dx.doi.org/10.1111/jonm.12654. PMid:30084198.

4. Silva Jr FF, Merino EA. Proposal for management of absenteeism among hospital nurses: a systematic review. Acta Paul Enferm. 2017;30(5):54653. http://dx.doi.org/10.1590/1982-0194201700079.

5. Oliveira PB, Spiri WC, Dell'Acqua MCQ, Mondini CCSD. Comparação entre ambiente de trabalho de hospitais públicos acreditado e não acreditado. Acta Paul Enferm. 2016 fev;29(1):53-9. http://dx.doi. org/10.1590/1982-0194201600008.

6. Santos JLG, Erdmann AL, Peiter CC, Alves MP, Lima SBS, Backes VMS. Comparison between the working environment of nurse managers and nursing assistants in the hospital context. Rev Esc Enferm USP. 2017;51:e03300. http://dx.doi.org/10.1590/s1980-220x2017017103300. PMid:29562043.

7. Santos RR, Paiva MCMS, Spiri WC. Associação entre qualidade de vida e ambiente de trabalho de enfermeiros. Acta Paul Enferm. 2018;31(5):472-9. http://dx.doi.org/10.1590/1982-0194201800067.

8. Dorigan $\mathrm{GH}$, Guirardello EB. Ambiente da prática, satisfação e clima de segurança: percepção dos enfermeiros. Acta Paul Enferm. 2017 abr;30(2):129-35. http://dx.doi.org/10.1590/1982-0194201700021.
9. Freitas RJM, Lima ECA, Vieira ÉS, Feitosa RMM, Oliveira GYM, Andrade LV. Stress of nurses in the urgency and emergency room. J Nurs UFPE on line. 2015;9(Suppl 10):1476-83. https://doi.org/10.5205/1981-8963v9i10a10861p1476-1483-2015.

10. Loro MM, Zeitoune RCG, Guido LA, Silveira CR, Silva RM. Revealing risk situations in the context of nursing work At urgency and emergency services. Esc Anna Nery. 2016;20(4):e20160086. http://dx.doi. org/10.5935/1414-8145.20160086.

11. Santos JLG, Lima MADS, Pestana AL, Colomé ICS, Erdmann AL. Strategies used by nurses to promote teamwork in an emergency room. Rev Gaúcha Enferm. 2016;37(1):e50178. http://dx.doi.org/10.1590/19831447.2016.01.50178. PMid:26934508.

12. Machado $\mathrm{MH}$, Aguiar Fo W, Lacerda WF, Oliveira E, Lemos W, Wermelinger $\mathrm{M}$ et al. Características gerais da enfermagem: o perfil sócio demográfico. Enferm. Foco. 2016;6(1/4):11-7. http://dx.doi. org/10.21675/2357-707X.2016.v7.nESP.686.

13. Lei $n^{\circ} 7.498$, de 25 de junho de 1986 (BR). Dispõe sobre a regulamentação do exercício da Enfermagem e dá outras providências. Diário Oficial da União [periódico na internet], Brasília (DF), 1986 [citado 10 fev 2019]. Disponível em: http://www.planalto.gov.br/ccivil_03/leis/l7498. htm

14. Mantovani VM, Nazareth JK, Keretzky KB, Maciel DNP, Biasibetti C Lucena AF et al. Research absenteeism due to illness among nursing professionals. Rev Min Enferm. 2015;19(3):641-6. http://dx.doi. org/10.5935/1415-2762.20150049.

15. Marcelino CF, Alves DFS, Gasparino RC, Guirardello EB. Validação do Nursing Work Index-Revised entre auxiliares e técnicos de enfermagem. Acta Paul Enferm. 2014 ago;27(4):305-10. http://dx.doi.org/10.1590/19820194201400052

16. Panunto MR, Guirardello EB. Professional nursing practice: environment and emotional exhaustion among intensive care nurses. Rev Lat Am Enfermagem. 2013;21(3):765-72. http://dx.doi.org/10.1590/S010411692013000300016. PMid:23918023.

17. Conselho Federal de Enfermagem. Pesquisa do Perfil dos Profissionais de Enfermagem no Brasil [Internet]. Brasília: COFEN;2015 [citado 2019 mar 2]. Disponível em: http://www.cofen.gov.br/perfilenfermagem/index. $\mathrm{html}$

18. Mantovani VM, Nazareth JK, Keretzky KB, Maciel DNP, Biasibetti C Lucena AF et al. Research absenteeism due to illness among nursing professionals. Rev Min Enferm. 2015;19(3):641-6. http://dx.doi. org/10.5935/1415-2762.20150049.

19. Brey C, Miranda FMD, Haeffner R, Castro IRS, Sarquis LMM, Felli VE. The absenteeism among health workers in a public hospital at south region of brazil. Revista de Enfermagem do CentroOeste Mineiro. 2017;7:e1135. http://dx.doi.org/10.19175/recom. v7i0.1135.

20. Santana LL, Sarquis LM, Brey C, Miranda FM, Felli VE. Absenteísmo por transtornos mentais em trabalhadores de saúde em um hospital no sul do Brasil. Rev Gaúcha Enferm. 2016;37(1):e53485. http://dx.doi. org/10.1590/1983-1447.2016.01.53485. PMid:27074306.

21. Santos JLG, Menegon FHA, De Pin SB, Erdmann AL, Oliveira RJT, Costa IAP. The nurse's work environment in a hospital emergency servisse. Rev Rene. 2017;18(2):195-203. http://dx.doi.org/10.15253/21756783.2017000200008.

22. Mudaly P, Nkosi ZZ. Factors influencing nurse absenteeism in a general hospital in Durban, South Africa. J Nurs Manag. 2015;23(5):623-31. http://dx.doi.org/10.1111/jonm.12189. PMid:24372737.

23. Ferro D, Zacharias FCM, Fabriz LA, Schonholzer TE, Valente SH Barbosa SM et al. Absenteísmo na equipe de enfermagem em serviços de emergência: implicações na assistência. Acta Paul Enferm. 2018 jul;31(4):399-408. http://dx.doi.org/10.1590/1982-0194201800056.

\footnotetext{
a Artigo extraído da tese "ASSOCIAÇÃO ENTRE AMBIENTE DE TRABALHO, ABSENTEÍSMO E EVENTOS ADVERSOS EM ENFERMEIROS E TÉCNICOS DE ENFERMAGEM DE UMA UNIDADE DE URGÊNCIA E EMERGÊNCIA", apresentada à Faculdade de Medicina, Universidade Estadual Paulista "Júlio de Mesquita Filho" no ano de 2020, autoria de Priscila Braga de Oliveira.
} 